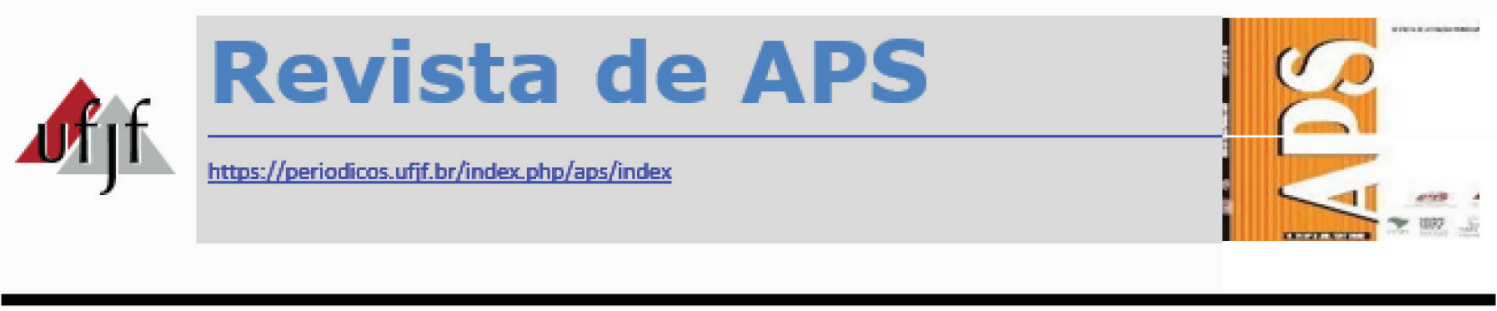

\title{
Grupo de autocuidado em hanseníase: benefícios na participação e resistências na adesão
}

\section{Self-care group in leprosy: benefits from participation and resistances to adherence}

\author{
Samara de Oliveira Barbosa ${ }^{1}$, Flávia Santos Medina ${ }^{2}$
}

\begin{abstract}
RESUMO
Objetivos: Descrever a limitação de atividades e a participação social de indivíduos com hanseníase antes e após participação em grupo de autocuidado, analisar os benefícios do grupo e a não adesão. Métodos: Trata-se de uma pesquisa descritiva e exploratória com abordagem quanti-qualitativa com 18 pacientes em tratamento para hanseníase, atendidos e acompanhados em um centro de saúde em Palmas Tocantins. Os dados foram coletados no período de março a dezembro de 2018 por meio de um Questionário de Identificação, da escala Screening of Activity Limitation and Safety Awareness (SALSA) e Participação Social, e Entrevista Semiestruturada. Os dados foram analisados pelo programa Statistical Package For Social Sciences (SPSS) e as entrevistas por meio de Análise de Conteúdo pela técnica análise categorial. Resultados: Embora a adesão ao grupo de autocuidado tenha sido baixa, a participação no mesmo contribuiu para redução dos escores das escalas Salsa e Participação Social e proporcionou aos pacientes conhecimento sobre a doença, troca de saberes, socialização, criação de vínculo, melhora da autonomia, do autocuidado e dos fatores emocionais. Conclusão: A limitação de atividades e a participação social dos pacientes podem ser prejudicadas pela hanseníase, entretanto, o grupo de autocuidado auxilia o paciente a lidar melhor com a doença.
\end{abstract}

PALAVRAS-CHAVE: Hanseníase. Autocuidado. Atividades Cotidianas. Participação Social.

\footnotetext{
${ }^{1}$ Enfermeira. Especialista em Saúde da Família e Comunidade na modalidade Residência Multiprofissional pelo Centro Universitário Luterano de Palmas - CEULP/ULBRA. Residente em Saúde Coletiva pelo CEULP/ULBRA. E-mail: sobarbosa1@gmail.com

${ }^{2}$ Fisioterapeuta. Mestre em Ciências da Saúde pela Universidade Federal do Tocantins - UFT. Filiação institucional Secretaria Municipal de Saúde de Palmas-TO.
} 


\begin{abstract}
Objectives: To describe the limitation of activities and social participation of individuals with leprosy before and after joining a self-care group, to analyze the benefits of the group and non-adherence. Methods: Descriptive and exploratory research with quantitative-qualitative approach with 18 patients undergoing treatment for leprosy, attended and followed up at a health center in Palmas - Tocantins. Data were collected from March to December 2018 through the Identification Questionnaire, SALSA Scales and Social Participation, and Semistructured Interview. The data were analyzed by the Statistical Package for Social Sciences (SPSS) Program and the interviews were conducted through Content Analysis by the categorical analysis technique. Results: Although adherence to the self-care group was low, the participation in it contributed to a reduction in the scores of the Salsa and Social Participation scales and provided patients with knowledge about the disease, knowledge exchange, socialization, improvement of autonomy, self-care and emotional factors. Conclusion: Patient's limitation of activities and social participation may be impaired by leprosy, however, the self-care group helps them to better deal with the disease.
\end{abstract}

KEYWORDS: Leprosy. Self Care. Activities of Daily Living. Social Participation.

\title{
INTRODUÇÃO
}

Segundo a Organização Mundial da Saúde (OMS), a hanseníase é uma doença infecciosa crônica causada pelo bacilo Mycobacterium leprae cuja multiplicação é lenta, o que pode levar de 1 a 20 anos para os sintomas serem manifestados. A pele, os nervos periféricos, a mucosa do trato respiratório superior e os olhos são os principais afetados. ${ }^{1}$

Dados de 2017 mostram que o Brasil ocupa o segundo lugar em casos novos no mundo com 26.875 casos, atrás da Índia com 126.164. ${ }^{2}$ Os estados brasileiros com maior número de casos novos em 2017 foram o Mato Grosso (3.431), Maranhão (3.104) e Pará (2.562). Em relação ao Tocantins (TO), este apresentou, em 2017, 1.241 casos novos e a segunda maior taxa de detecção com 80,05 por 100.000 habitantes, atrás do Mato Grosso com 102,58. A capital, Palmas, apresentou 526 casos novos no mesmo ano ${ }^{3}$.

A hanseníase, se não diagnosticada precocemente, pode ser incapacitante e gerar muitas mudanças na vida dos pacientes. ${ }^{4}$ As incapacidades e deformidades da hanseníase podem causar limitações aos pacientes e dificultar que estes sejam ativos na sociedade, interferindo na capacidade de trabalho, na vida social e familiar, resultando em problemas psicológicos relacionados ao estigma e preconceito que podem isolá-los do convívio social. ${ }^{5,6}$ O Ministério da Saúde sugere, por meio dos manuais de autocuidado, que ações educativas com esse foco sejam realizadas para prevenir as incapacidades. 0 autocuidado minimiza e/ou previne os efeitos da hanseníase. A aderência às orientações do autocuidado demanda informação e conceitos que levam o paciente a compreender 
as possíveis alterações na imagem corporal devido à doença. Dessa forma, o paciente com orientações adequadas e conhecimento sobre a doença pode reconhecer sua dor, angústia, esperanças e perspectivas. ${ }^{7}$ Torna-se necessário ter um diálogo entre a pessoa com hanseníase e a equipe multiprofissional para que reconheçam a importância do autocuidado. ${ }^{8} \mathrm{~A}$ desestruturação da imagem corporal, o estigma do corpo leproso, o desconhecimento da doença, a capacidade de autocuidado e o não compromisso com a saúde são alguns fatores que dificultam os pacientes a realizarem o autocuidado., ${ }^{9,7}$

Consoante a isso, os grupos de autocuidado em hanseníase são instrumentos que incorporam saberes e práticas e visam à superação das limitações, proporcionam interação entre os participantes e troca de experiências e fortalecem a autonomia dos indivíduos. Esses grupos capacitam a pessoa acometida pela doença a conhecer os riscos para sua integridade física e a identificar o problema visando a solução, e principalmente, a adesão ao autocuidado. ${ }^{10}$ Portanto, a equipe de saúde deve possuir uma visão integral da pessoa com hanseníase e ter, além do cuidado técnico, a preocupação com o fator psíquico e sociocultural do cuidado na hanseníase. ${ }^{7}$ Diante disso, é necessária a avaliação da limitação para realizar atividades do cotidiano e da participação social para analisar o quanto a doença está interferindo na vida dos pacientes e nortear as ações dos profissionais da saúde para melhoria da qualidade de vida daqueles. As escalas são ferramentas potentes para avaliar o progresso das oficinas do grupo de autocuidado. Assim, definiram-se como objetivos do estudo descrever a limitação de atividades e a participação social de indivíduos com hanseníase antes e após participação em grupo de autocuidado, analisar os benefícios do grupo e a não adesão.

\section{MÉTODOS}

Trata-se de uma pesquisa descritiva e exploratória com abordagem quantiqualitativa com 18 pacientes em tratamento para hanseníase, atendidos e acompanhados em um Centro de Saúde da Comunidade, localizado em Palmas - Tocantins, realizada no período de março a dezembro de 2018. Esse centro de saúde é uma Unidade Básica de Saúde (UBS), composta por quatro equipes de Estratégia de Saúde da Família (ESF) e uma equipe de Núcleo Ampliado de Saúde da Família e Atenção Básica (NASF-AB).

A população foi constituída por usuários que participaram de pelo menos quatro encontros do grupo de autocuidado e por usuários que não participaram em nenhum momento do grupo. Os critérios de inclusão foram: pacientes com idade superior a 18 anos, em tratamento para hanseníase, que possuíam condições cognitivas para responder as escalas e a entrevista, e aceitaram participar do estudo por meio da assinatura do Termo de Consentimento Livre e Esclarecido (TCLE). Os critérios de exclusão foram: pacientes que abandonaram o tratamento para hanseníase, residentes fora da área de abrangência da UBS ou em outro município. 
Os instrumentos utilizados para coleta de dados foram: um Questionário de Identificação, a escala Screening of Activity Limitation and Safety Awareness (SALSA) e a escala de Participação Social.

O Questionário de Identificação é composto por questões referentes à idade, sexo, estado civil, escolaridade, ocupação, classificação operacional da hanseníase, forma clínica, grau de incapacidade, escore OMP (olhos, mãos, pés), presença de reação hansênica e data de participação no grupo de autocuidado. O instrumento foi elaborado pelas autoras da pesquisa e preenchido com base nas respostas do paciente e dados do prontuário físico e eletrônico.

Com base na Classificação Internacional de Funcionalidade, Incapacidade e Saúde (CIF), realizou-se esforço internacional para a elaboração de dois instrumentos: a SALSA (Screening Activity Limitation and Safety Awareness), para medir limitação de atividade e consciência de risco, e a Escala de Participação Social, para medir restrição de participação. As duas escalas são baseadas em questionários e são recomendadas pelo Ministério da Saúde para que sejam utilizadas nos serviços de saúde do Brasil, podendo ser aplicadas pelo menos no início e no final do tratamento e também como parâmetro para avaliar resultados de intervenções realizadas, como os grupos de autocuidado. ${ }^{11,12}$

A escala SALSA objetiva avaliar a limitação de atividade, ou seja, o quanto a pessoa consegue realizar suas atividades de vida diária, e o risco de se aumentar as deficiências durante a realização dessas atividades. ${ }^{11}$ É um instrumento padronizado que contem 20 itens dentro dos domínios de mobilidade, autocuidado, trabalho e destreza. ${ }^{13,14}$ Conceitualmente o escore varia de 0 a 80 , mas em cinco países nos quais se entrevistaram 568 pessoas, os escores variaram entre 10 a 80 em pacientes com hanseníase ou diabetes. ${ }^{11}$ Dessa forma, escore baixo indica pouca dificuldade relacionada às atividades diárias, já os mais altos indicam maior limitação de atividade. ${ }^{13}$

Outro item avaliado na escala SALSA é a consciência de risco, esta consiste em identificar e analisar a capacidade do indivíduo em perceber o risco que corre ao realizar certas atividades. ${ }^{13,14}$ Seu escore varia entre 0 e 11 . Assim sendo, escores mais altos indicam uma consciência elevada dos riscos em certas atividades, entretanto, em consequência, indicam que há uma limitação de atividade. ${ }^{11}$

O desenvolvimento da escala SALSA foi realizado por alguns países, dentre eles o Brasil, e recebeu apoio financeiro das instituições American Leprosy Mission (ALM) e The Leprosy Mission Internacional (TLMI). A escala foi finalizada em 2004, revista em 2005, 2006 e 2008, a partir de feedback recebido. ${ }^{11}$

A escala de Participação Social versão 6.0 contém 18 itens cuja somatória dos pontos varia de 0 a 90, sendo os valores de 0 a 12 considerados sem restrições significativas à participação social. Essa escala objetiva avaliar o aprendizado e aplicação do conhecimento; comunicação e cuidados pessoais; mobilidade; vida doméstica; 
interações e relacionamentos interpessoais e em comunidade; principais áreas da vida e comunidade. ${ }^{15,16}$ Para responder à escala, solicita-se que o entrevistado pense em alguém semelhante, mas sem doença ou deficiência, e se compare a ele. ${ }^{11}$

Um dos princípios que foram estabelecidos para a criação das escalas SALSA e de Participação Social é que estas são transculturais e por isso foram elaboradas simultaneamente em vários países. ${ }^{11} \mathrm{~A}$ Escala de Participação foi validada mediante pesquisa multicêntrica na Índia, Brasil e Nepal. ${ }^{14}$ Além de versão em português e validadas no Brasil, as duas escalas estão sendo aplicadas em diversos cenários socioculturais. ${ }^{17}$

O grupo de autocuidado em hanseníase foi implantado em março de 2018, sendo os encontros, em geral, realizados quinzenalmente e em horário noturno, com duração de aproximadamente duas horas cada. A escolha do horário foi em razão da disponibilidade da maioria dos pacientes. Nas reuniões do grupo participaram e contribuíram com seus conhecimentos: enfermeiro, dentista, psicólogo, fisioterapeuta, nutricionista e assistente social.

Os pacientes foram avaliados após primeira participação no grupo de autocuidado e após quarta participação. Também foi utilizado como instrumento para coleta de dados um roteiro de entrevista semiestruturada que questionou quanto aos benefícios obtidos com a participação no grupo. A entrevista foi realizada após o quarto dia de participação e proporcionando ao entrevistado liberdade de expressão em suas respostas, pois através da entrevista "o pesquisador organiza um conjunto de questões sobre o tema que está sendo estudado, mas permite e às vezes até incentiva, que o entrevistado fale livremente sobre assuntos que vão surgindo com o desdobramento do tema principal". 18:70

Para os pacientes que não tiveram interesse em participar do grupo, foi elaborado um roteiro de entrevista que questionou quanto aos motivos do desinteresse. Todas as entrevistas, após consentimento, foram gravadas e transcritas.

O grupo de autocuidado em hanseníase foi criado no centro de saúde supracitado e logo depois os pacientes foram convidados a participar por meio das consultas, telefonemas e visitas domiciliares. As oficinas do grupo foram baseadas nos manuais e nas cartilhas propostas para as ações educativas em autocuidado elaboradas pelo Ministério da Saúde, como o Guia de apoio para grupos de autocuidado em hanseníase ${ }^{10}$, autocuidado em hanseníase: face, mãos e pés ${ }^{19}$, Eu me cuido e vivo melhor ${ }^{20}$, aspectos da doença e seus fatores emocionais e sociais também foram discutidos. As oficinas foram realizadas pela pesquisadora responsável deste estudo com apoio dos profissionais da ESF e do NASF-AB, por meio de roda de conversa, dinâmicas, exposição de vídeos e distribuição de material educativo.

Para coleta de dados, os pacientes foram abordados após participarem do grupo e/ou em visita domiciliar agendada. O controle da participação dos pacientes no grupo foi realizado a partir de uma lista de frequência assinada por eles em todos os encontros. Após a coleta, os dados foram exportados e analisados descritivamente pelo 
Microsoft Office Excel versão 2013. Para verificar a relação estatística entre as variáveis "limitação de atividade" e "participação social", foi realizado o teste de correlação de Pearson, utilizando o software Statistical Package For Social Sciences (SPSS), versão 20.0, considerado um nível de significância de $\leq 0,05$.

A análise das duas entrevistas foi realizada a partir da análise de conteúdo pela técnica análise categorial proposta por Bardin. ${ }^{21} \mathrm{Em}$ seguida foi realizada a conclusão da análise e dos resultados obtidos.

O projeto foi aprovado pela Secretaria Municipal de Saúde de Palmas-TO com no 94 de 12/2017 e pelo Comitê de Ética e Pesquisa do Centro Universitário Luterano de Palmas/ Universidade Luterana do Brasil (CEULP/ULBRA) com parecer nํ 2.909.504/2018 e CAAE no 83724418.7.0000.5516/2018, de acordo com a Resolução do Conselho Nacional de Saúde no 466/12 que normatiza pesquisas envolvendo seres humanos. ${ }^{22}$

\section{RESULTADOS}

A princípio, o Centro de Saúde contava com aproximadamente 30 pacientes em tratamento para hanseníase, entretanto, apesar do convite a todos, somente 10 participaram do grupo no período desse estudo. Além desses, também participaram do grupo cinco pacientes que já haviam concluído o tratamento e um que não era da área de abrangência do centro de saúde. Após iniciado o grupo, dos 10 pacientes em tratamento que estavam dentro dos critérios da pesquisa, quatro deixaram de comparecer às reuniões, um por licença-maternidade, um por mudança de residência e tratamento em outro centro de saúde e dois por erro de diagnóstico, sendo, portanto, excluídos do estudo.

Dessa forma, a população final deste estudo foi de 18 pacientes em tratamento para hanseníase, sendo que somente 6 participaram do grupo de autocuidado pelo menos quatro vezes. Os demais, 12 indivíduos, participaram da pesquisa respondendo quanto aos motivos do não interesse no grupo.

Em relação ao perfil dos pacientes participantes do grupo, a faixa etária variou de 40 a 71 anos, $4(66,66 \%)$ eram do sexo masculino, 5 (83,33\%) eram casados e tinham apenas ensino fundamental, 2 (33,33\%) eram aposentados. Todos os participantes do grupo tinham classificação operacional multibacilar e forma clínica dimorfa, 5 (83,33\%) tinham grau 1 de incapacidade, 3 (50\%) tinham soma OMP (olhos, mãos e pés) e 3 (50\%) não tinham reação hansênica (Tabela 1). 
Tabela 1 - Perfil sociodemográfico e clínico dos pacientes participantes do grupo de autocuidado

\begin{tabular}{|c|c|c|c|c|}
\hline \multirow[b]{2}{*}{ Variáveis } & \multicolumn{2}{|c|}{ Sexo } & \multirow[b]{2}{*}{ Total } & \multirow[b]{2}{*}{ \%Total } \\
\hline & Feminino & Masculino & & \\
\hline \multicolumn{5}{|l|}{ Faixa Etária } \\
\hline $40-49$ & 2 & - & 2 & $33,33 \%$ \\
\hline $50-59$ & - & 2 & 2 & $33,33 \%$ \\
\hline $60-69$ & - & 1 & 1 & $16,67 \%$ \\
\hline $\begin{array}{l}70-79 \\
\text { Estado Civil }\end{array}$ & - & 1 & 1 & $16,67 \%$ \\
\hline Casado & 2 & 3 & 5 & $83,33 \%$ \\
\hline Solteiro & - & 1 & 1 & $16,67 \%$ \\
\hline \multicolumn{5}{|l|}{ Escolaridade } \\
\hline Ensino Fundamental & 2 & 3 & 5 & $83,33 \%$ \\
\hline Ensino Médio & - & 1 & 1 & $16,67 \%$ \\
\hline \multicolumn{5}{|l|}{ Ocupação } \\
\hline Aposentado & - & 2 & 2 & $33,33 \%$ \\
\hline Autônomo & - & 1 & 1 & $16,67 \%$ \\
\hline Aux. Serviços Gerais & 1 & - & 1 & $16,67 \%$ \\
\hline Cozinheira & 1 & - & 1 & $16,67 \%$ \\
\hline Vigilante & - & 1 & 1 & $16,67 \%$ \\
\hline \multicolumn{5}{|c|}{ Classificação Operacional } \\
\hline $\begin{array}{l}\text { Multibacilar } \\
\text { Forma Clínica }\end{array}$ & 2 & 4 & 6 & $100,00 \%$ \\
\hline Dimorfa & 2 & 4 & 6 & $100,00 \%$ \\
\hline \multicolumn{5}{|l|}{ Grau de Incapacidade } \\
\hline 1 & 2 & 3 & 5 & $83,33 \%$ \\
\hline 0 & - & 1 & 1 & $16,67 \%$ \\
\hline \multicolumn{5}{|l|}{ OMP } \\
\hline 0 & - & 1 & 1 & $16,67 \%$ \\
\hline 1 & 1 & 1 & 2 & $33,33 \%$ \\
\hline 2 & 1 & 2 & 3 & $50,00 \%$ \\
\hline \multicolumn{5}{|l|}{ Reação } \\
\hline Não & 1 & 2 & 3 & $50,00 \%$ \\
\hline Neurite Isolada & - & 1 & 1 & $16,67 \%$ \\
\hline Tipo 1 & 1 & 1 & 2 & $33,33 \%$ \\
\hline Total & 2 & 4 & 6 & $100,00 \%$ \\
\hline
\end{tabular}

Fonte: elaborada pelas autoras

No presente estudo, os escores de limitação de atividades variaram de 20 a 42 pontos no primeiro dia de participação no grupo e de 19 a 28 após o quarto dia, o que significa que houve uma redução nos escores. Na primeira participação, 3 pacientes (50\%) apresentaram leve limitação, 2 (33,3\%) sem limitação e 1 (16,6\%) moderada limitação. Após o quarto dia de participação, 5 (83,3\%) apresentaram-se sem limitação e $1(16,6 \%)$ com leve limitação (Tabela 2 ).

Em relação à avaliação da consciência de risco, os escores variaram de 0 a 3 pontos na primeira participação e de 0 a 1 após a quarta, o que significa que os pacientes diminuíram sua consciência nos riscos das atividades, mas por outro lado 
significa redução do escore SALSA e, consequentemente, melhora do nível da limitação de atividade (Tabela 2).

Tabela 2 - Escala SALSA na primeira e após a quarta participação no grupo de autocuidado

\begin{tabular}{|c|c|c|c|c|c|c|c|}
\hline $\begin{array}{l}\text { Faixa } \\
\text { Etária }\end{array}$ & Sexo & $\begin{array}{l}\text { Escore } \\
\text { SALSA1 }\end{array}$ & $\begin{array}{l}\text { Escore } \\
\text { SALSA4 }\end{array}$ & CR1 & CR4 & $\begin{array}{c}\text { SALSA na 1à } \\
\text { participação no } \\
\text { grupo }\end{array}$ & $\begin{array}{l}\text { SALSA após 4a } \\
\text { participação no } \\
\text { grupo }\end{array}$ \\
\hline \multirow{2}{*}{$40-49$} & \multirow{2}{*}{$\mathrm{F}$} & 20 & 20 & 0 & 0 & Sem Limitação & Sem Limitação \\
\hline & & 42 & 28 & 2 & 1 & Moderada Limitação & Leve Limitação \\
\hline \multirow{2}{*}{$50-59$} & \multirow{2}{*}{ M } & 24 & 19 & 1 & 0 & Sem Limitação & Sem Limitação \\
\hline & & 30 & 22 & 2 & 1 & Leve Limitação & Sem Limitação \\
\hline $60-69$ & M & 32 & 23 & 2 & 0 & Leve Limitação & Sem Limitação \\
\hline $70-79$ & $M$ & 31 & 24 & 3 & 1 & Leve Limitação & Sem Limitação \\
\hline Média & & 29,8 & 22,7 & 1,7 & 0,5 & - & - \\
\hline \multicolumn{2}{|c|}{ Desvio Padrão } & 7,5 & 3,2 & 1,0 & 0,5 & - & - \\
\hline
\end{tabular}

F - Feminino; M - Masculino; CR - Consciência de Risco

Fonte: elaborada pelas autoras

Na escala de participação social (Tabela 3), a presente pesquisa mostra que 4 (66,66\%) participantes não apresentaram restrição significativa, $1(16,66 \%)$ apresentou leve restrição e 1 (16,66\%) restrição moderada na primeira participação no grupo. Após a quarta participação, 5 (83,33\%) participantes não tiveram restrição significativa. Os escores da participação social variaram de 0 a 27 no primeiro dia e de 0 a 19 após o quarto dia de participação.

Tabela 3 - Escala de Participação Social na primeira e após a quarta participação no grupo de autocuidado

\begin{tabular}{|c|c|c|c|c|c|}
\hline $\begin{array}{l}\text { Faixa } \\
\text { Etária }\end{array}$ & Sexo & $\begin{array}{l}\text { Escore } \\
\text { PS1 }\end{array}$ & $\begin{array}{l}\text { Escore } \\
\text { PS4 }\end{array}$ & $\begin{array}{c}\text { Participação Social no } \\
19 \text { grupo }\end{array}$ & $\begin{array}{l}\text { Participação Social } \\
\text { após } 4 \text { ㅇ grupo }\end{array}$ \\
\hline \multirow{2}{*}{$40-49$} & \multirow{2}{*}{$\mathrm{F}$} & 0 & 0 & $\begin{array}{l}\text { Sem Restrição } \\
\text { Significativa }\end{array}$ & $\begin{array}{l}\text { Sem Restrição } \\
\text { Significativa }\end{array}$ \\
\hline & & 2 & 0 & $\begin{array}{l}\text { Sem Restrição } \\
\text { Significativa }\end{array}$ & $\begin{array}{l}\text { Sem Restrição } \\
\text { Significativa }\end{array}$ \\
\hline \multirow{2}{*}{$50-59$} & \multirow{2}{*}{$M$} & 15 & 8 & Leve Restrição & $\begin{array}{l}\text { Sem Restrição } \\
\text { Significativa }\end{array}$ \\
\hline & & 8 & 2 & $\begin{array}{l}\text { Sem Restrição } \\
\text { Significativa }\end{array}$ & $\begin{array}{l}\text { Sem Restrição } \\
\text { Significativa }\end{array}$ \\
\hline $60-69$ & $M$ & 27 & 19 & Restrição Moderada & Leve Restrição \\
\hline $70-79$ & $M$ & 3 & 3 & $\begin{array}{l}\text { Sem Restrição } \\
\text { Significativa }\end{array}$ & $\begin{array}{l}\text { Sem Restrição } \\
\text { Significativa }\end{array}$ \\
\hline \multicolumn{2}{|c|}{ Média } & 9,2 & 5,3 & - & - \\
\hline \multicolumn{2}{|c|}{ Desvio Padrão } & 10,3 & 7,3 & - & - \\
\hline
\end{tabular}

F- Feminino; M - Masculino; PS - Participação Social

Fonte: elaborada pelas autoras 
De acordo com o coeficiente de correlação de Pearson $(0,11$ e - -0,02) e o valor de $P(0,84$ e 0,97$)$, verificou-se que não houve relação entre as variáveis nos dois momentos estudados.

Abaixo, encontram-se os benefícios obtidos pelos seis pacientes após a participação no grupo, relatados por meio da entrevista semiestruturada.

\section{Benefícios da participação no grupo}

Nessa categoria foi possível identificar por meio dos relatos que os benefícios foram conhecimento sobre a doença, criação de vínculo entre participantes e troca de experiências, autocuidado, diminuição do preconceito e estigma.

A maioria dos entrevistados afirmou como principal benefício o conhecimento sobre a doença, que foi fundamental para saber lidar com a mesma, sanar as dúvidas e identificar cuidados que devem adotar, como mostram os seguintes discursos:

"A gente não tinha conhecimento com a doença, mas depois que eu vim para o grupo aí a gente teve mais aberto, saber que não é assim tão difícil, né?! Eu fiquei mais alegre porque eu estava assim: - Nossa, mas eu peguei essa doença?! O quê que é isso será?! Sinto que conversando com vocês, com as pessoas, é uma doença que tem cura e não precisa ter medo né?! Porque aí tirou essas dúvidas de mim. Que a gente (ele e esposa) não sabia muito assim como que ia fazer, como ia começar (P1)."

"Eu aprendi a saber várias coisas sobre a doença também. Teve benefícios porque aprendi mais sobre a doença e sei como lidar com ela (P2)."

"Mudou muitas coisas na minha vida porque eu não sabia como se dava com ela (hanseníase), entendeu?! Eu participando do grupo eu aprendi como que se dá, o quê que eu tenho que fazer, o que eu não tenho. É isso! Antigamente eu não sabia né?! (P3)."

Os participantes criaram vínculo com os profissionais e com os outros pacientes em tratamento que faziam parte do grupo de autocuidado. Houve troca de experiências, cada um relatando seus sentimentos e vivências.

"Conheci outras pessoas, a gente falou dos problemas que a gente tem (P3)."

"É mais um contato assim com a enfermeira, tá conversando com ela normalmente. Então o benefício maior foi esse, porque sempre a enfermeira, o pessoal, tão sempre mais por fora né?! (P5)."

"O quê que melhorou? Assim, a convivência com as pessoas e saber que a hanseníase tem cura [...] (P6)."

Alguns pacientes relataram que melhoraram o autocuidado a partir das participações no grupo e realizaram os exercícios propostos, na busca de finalizarem bem o tratamento da doença. 


\begin{abstract}
"Depois que comecei a vir nas reuniões eu comecei a me cuidar mais direito né?! Tomar mais cuidado pra ficar melhor, pra terminar o tratamento bem (P3)."

"Ainda hoje eu tava ali na frente, tava lembrando dos exercícios das mãos. É isso que são os benefícios que a gente consegue pegar. Às vezes pego até sem querer, eu lembro e já começo a fazer (P4)."
\end{abstract}

Um participante afirma que o conhecimento adquirido no grupo é importante para diminuir o preconceito, no entanto observou-se que alguns pacientes pareciam não acreditar que tinham realmente a doença e buscavam saber como foi e quem os "contaminou". Evidenciou-se o preconceito e o estigma que existe em relação à hanseníase:

\begin{abstract}
"[...] saber que a hanseníase tem cura [...]. Pra mim foi importante porque acabou esse preconceito da doença, né?!. O grupo melhorou muito o entendimento e a pessoa entender que não é assim esse preconceito e acabar o preconceito que existe sobre as pessoas que tem hanseníase. Pra mim isso ai foi importante, o melhor, a conversa, as reuniões, discutindo sobre esses aspectos (P6)."
\end{abstract}

"A busca minha é de onde me contaminei, é a principal busca. Vim para o grupo para isso, para saber das coisas e infelizmente isso aí eu não consegui saber, como é que eu fui contaminado. Onde? Quando? Não consegui. Isso é meu objetivo principal e porque a partir dai eu ia tomar mais cuidado dentro da família pra saber e pra evitar coisas (P4)."

“Eu não sei nem como é que essa doença apareceu em mim, porque eu nunca senti nada (P5)."

Em relação ao perfil dos 12 pacientes que não participaram do grupo de autocuidado, a faixa etária variou de 22 a 86 anos, 7 (58,33\%) são do sexo feminino e 6 (50\%) eram casados. Quanto à escolaridade, 4 (33,33\%) tinham ensino superior, 4 (33,33\%) ensino médio, 4 (33,33\%) ensino fundamental. A ocupação foi descrita como autônoma por seis (50\%) pacientes. Todos os que não participaram do grupo tinham classificação operacional multibacilar e forma clínica dimorfa, 7 (58,33\%) tinham grau de incapacidade 0 e soma OMP 0 , sendo que nenhum apresentou reação hansênica (Tabela 4). 
Tabela 4 - Perfil sociodemográfico e clínico dos pacientes que não participaram do grupo de autocuidado

\begin{tabular}{|c|c|c|c|c|}
\hline \multirow{2}{*}{ Variáveis } & \multicolumn{2}{|c|}{ Sexo } & \multirow{2}{*}{ Total } & \multirow{2}{*}{ \%Total } \\
\hline & Feminino & Masculino & & \\
\hline \multicolumn{5}{|l|}{ Faixa Etária } \\
\hline $20-29$ & 2 & 1 & 3 & $25,00 \%$ \\
\hline $30-39$ & 2 & - & 2 & $16,67 \%$ \\
\hline $50-59$ & 1 & 1 & 2 & $16,67 \%$ \\
\hline $60-69$ & 2 & 2 & 4 & $33,33 \%$ \\
\hline $80-89$ & - & 1 & 1 & $8,33 \%$ \\
\hline \multicolumn{5}{|l|}{ Estado Civil } \\
\hline Casado & 4 & 2 & 6 & $50,00 \%$ \\
\hline Divorciado & - & 1 & 1 & $8,33 \%$ \\
\hline Solteiro & 3 & 2 & 5 & $41,67 \%$ \\
\hline \multicolumn{5}{|l|}{ Escolaridade } \\
\hline Ensino Fundamental & 2 & 2 & 4 & $33,33 \%$ \\
\hline Ensino Médio & 2 & 2 & 4 & $33,33 \%$ \\
\hline Ensino Superior & 3 & 1 & 4 & $33,33 \%$ \\
\hline \multicolumn{5}{|l|}{ Ocupação } \\
\hline Aposentado & 1 & 1 & 2 & $16,67 \%$ \\
\hline Atendente de Loja & 1 & - & 1 & $8,33 \%$ \\
\hline Autônomo & 4 & 2 & 6 & $50,00 \%$ \\
\hline Dona de Casa & 1 & - & 1 & $8,33 \%$ \\
\hline Funcionário Público & - & 1 & 1 & $8,33 \%$ \\
\hline Mecânico & - & 1 & 1 & $8,33 \%$ \\
\hline \multicolumn{5}{|c|}{ Classificação Operacional } \\
\hline Multibacilar & 7 & 5 & 12 & $100,00 \%$ \\
\hline \multicolumn{5}{|l|}{ Forma clínica } \\
\hline Dimorfa & 7 & 5 & 12 & $100,00 \%$ \\
\hline \multicolumn{5}{|l|}{ Grau de Incapacidade } \\
\hline 1 & 2 & 3 & 5 & $41,67 \%$ \\
\hline 0 & 5 & 2 & 7 & $58,33 \%$ \\
\hline \multicolumn{5}{|l|}{ OMP } \\
\hline 0 & 5 & 2 & 7 & $58,33 \%$ \\
\hline 1 & - & 1 & 1 & $8,33 \%$ \\
\hline 2 & 1 & 1 & 2 & $16,67 \%$ \\
\hline 3 & 1 & - & 1 & $8,33 \%$ \\
\hline 4 & - & 1 & 1 & $8,33 \%$ \\
\hline \multicolumn{5}{|l|}{ Reação } \\
\hline Não & 7 & 5 & 12 & $100,00 \%$ \\
\hline Total & 7 & 5 & 12 & $100,00 \%$ \\
\hline
\end{tabular}

Fonte: elaborada pelas autoras

Abaixo, encontram-se os motivos relatados pelos pacientes para a não participação no grupo de autocuidado, obtidos por meio da entrevista semiestruturada. 


\section{Motivos para a não participação no grupo}

Os entrevistados destacaram como motivos de não participarem do grupo a falta de tempo, o preconceito e estigma, fatores psicológicos, mal-estar e falta de interesse. A maioria dos entrevistados relata que não participa do grupo devido à falta de tempo em razão dos horários de trabalho, obrigação de cuidar de netos e outros compromissos assumidos, conforme se nota nas falas:

"Por falta de tempo né?! Assim, oportunidade mesmo e questão de horário, distância e tal. Fico mais tempo em chácara. Por isso (P7)."

"Eu não participo devido os horários, devido também que não bate com o meu, porque eu trabalho dia todo e geralmente faço plantão a noite. Ai já tenho outro emprego e não tá batendo compatível com os horários (P15)."

"Porque sempre nessas épocas que você me chamou eu tava trabalhando fora. Eu saio às vezes fora do estado (P8)."

"Eu tenho outro compromisso nos dias do grupo. Eu tenho consciência de que a participação nessas rodas de conversas é de suma importância, mas infelizmente todas as vezes que você me convidou eu também tinha outros compromissos que não pude deixar de comparecer (P9)."

"Eu fico numa chácara cuidando da minha neta porque minha filha trabalha (P17)."

Um entrevistado destaca que não participa devido à vergonha, medo de que outras pessoas descubram que está em tratamento para hanseníase, devido ao preconceito com a doença, e que isso interfira em seu trabalho.

"Eu dei um jeito de me adaptar só que ai eu fiquei com vergonha. Porque é uma coisa que tem muito preconceito né?! E ai as pessoas ficam vendo e como eu trabalho nesse ramo [faz e vende salgados]. Aí, aí fulano faz isso, então... entendeu?! Eu já vim e voltei daqui. Tudo por causa da vergonha. Preconceito tu sabe que ainda existe demais e pelo fato assim: vai que tem alguém que conhece e sabe com quê que eu trabalho e aí não vai entender que depois que você inicia o tratamento não tem mais esse risco. Mas nem todo mundo tem esse conhecimento e entende, aí fica naquela: Ah...! Então por isso eu preferi não participar (P13)."

Outra entrevistada relata desânimo, dor no corpo e mal-estar, mas não sabe se tem relação com a doença ou se é devido ao envelhecimento.

"Tem dias que tô desanimada, não tenho vontade de sair de casa, dá aquele desânimo! Tem horas que minha menina chega lá em casa e chama pra sair e eu ponho tudo difícil e não vou. - Ah estou com o corpo doendo, tô com mal-estar e acabo não indo. Não tenho vontade de ir para o grupo, mas não sei se pela doença que faz isso ou idade também. Às vezes porque nunca vim. Se eu viesse um dia, quem sabe (P14)." 
Percebeu-se o desinteresse de um paciente em participar do grupo em razão de o mesmo possuir conhecimento sobre o autocuidado, incluindo exercícios que deveriam ser realizados.

\section{DISCUSSÃO}

A literatura traz que a maioria dos pacientes em tratamento para hanseníase é do sexo masculino ${ }^{23,24}$. Os dados nacionais, estaduais e municipais também confirmam essa predominância, com 55,4\%, 54,3\% e 54,7\%, respectivamente, segundo dados do DATASUS de 2017. ${ }^{3}$ Neste estudo a porcentagem de participantes do sexo masculino foi um pouco inferior aos dados apresentados, pois correspondeu à metade da amostra.

Estudo realizado com 32 pacientes com hanseníase teve como resultado que $59,4 \%$ eram do sexo masculino, a classificação operacional multibacilar foi mais frequente em (81,3\%), assim como a forma clínica não classificada $(43,8 \%)$ seguida da forma dimorfa $(28,1 \%){ }^{23}$ De forma semelhante, uma pesquisa com 129 pacientes em tratamento da hanseníase demonstrou que o sexo masculino predominou (62\%), a classificação operacional multibacilar foi mais prevalente com 102 pacientes (79,05\%), mas a forma clínica virchoviana foi a mais presente em 60 (46,51\%) indivíduos, seguida pela dimorfa em $28(21,71 \%)$ pacientes. ${ }^{24} \quad \mathrm{Na}$ presente pesquisa verificou-se que a totalidade dos casos eram multibacilares na população estudada. No Brasil, quase $75 \%$ dos casos foram classificados como multibacilares em 2017, o que demonstra que os dados deste estudo são bem superiores aos nacionais, o que pode indicar diagnóstico tardio no serviço de saúde local. ${ }^{3}$

Nesta pesquisa, em relação ao total de participantes, sobressaiu o grau de incapacidade 1 (10 participantes), diferente do estudo que teve como objetivo traçar o perfil dos portadores de hanseníase de um Centro de Referência em Dermatologia em que foram observados 469 prontuários de pacientes, e destes, a maioria $(66,7 \%)$ apresentou grau de incapacidade zero na primeira avaliação. ${ }^{25}$

O Grau de Incapacidade Física é um indicador epidemiológico que identifica pacientes com maiores chances de terem reações e novas incapacidades. O Escore OMP é a somatória dos graus de incapacidades de cada segmento e representa uma ferramenta que proporciona mais precisão na classificação do comprometimento dos diferentes seguimentos, o que viabiliza um melhor cuidado ao paciente. ${ }^{26}$

Em outro estudo realizado no Rio de Janeiro em que foram observados 667 prontuários de pacientes com hanseníase, 377 (56,52\%) deles não apresentaram reação hansênica. Os dados da presente pesquisa apresentam porcentagem maior de pacientes, em sua totalidade, sem reação hansênica (83,33\%). É necessário estar atento para o rápido diagnóstico e cuidado da reação hansênica em razão de ela ser a maior causadora de lesão no nervo periférico e incapacidades. ${ }^{27}$ 
Em pesquisa realizada com 17 pacientes de hanseníase participantes de um grupo de autocuidado, em que foram aplicadas as escalas SALSA e de Participação Social, observou-se que os escores de limitação de atividades variaram de 20 a 44 pontos, sendo que $72 \%$ tiveram leve limitação, 14\% mostraram-se sem limitação e 14\% com limitação moderada. Em relação à escala de Participação Social, $43 \%$ da amostra não tiverem restrição, 29\% apresentaram leve restrição, 14\% com moderada restrição e 14 \% com grande restrição, corroborando com os resultados do presente estudo. ${ }^{14} \mathrm{Da}$ mesma forma, um estudo executado com aplicação da escala de Participação Social em 20 pacientes em tratamento para hanseníase em Juiz de Fora-MG mostrou que $60 \%$ deles foram classificados como sem restrição significativa. ${ }^{28}$ Os autores do estudo consideram que "a ausência de restrição pode ser justificada em grande parte pela ausência de sequelas causadas pela hanseníase". 28:250

Estudo realizado em pessoas com hanseníase na Indonésia demonstrou que cerca de $60 \%$ dos pacientes relataram algum grau de limitação na execução de atividades diárias e restrição de participação social. A gravidade do comprometimento da doença e nível de escolaridade, atividade e estigma foram os determinantes da participação social. ${ }^{29}$

$\mathrm{Na}$ pesquisa efetuada com 54 pacientes com pós-alta medicamentosa para tratamento de hanseníase em São José do Rio Preto, a análise da escala SALSA evidenciou que $57,4 \%$ dos participantes da pesquisa tiveram algum grau de limitação das atividades, sendo 31,5\% leve limitação, 18,5\% moderada limitação, 7,4\% muito severa limitação e 42,6 \% não apresentaram nenhuma limitação. ${ }^{30}$

Estudo executado em Sobral-CE com objetivo de caracterizar a limitação funcional, de atividade, consciência de risco e restrição à participação social em 69 pessoas atingidas pela hanseníase no pós-alta mostrou que 64 (92,8\%) indivíduos não apresentaram nenhuma restrição significativa à participação social, 4 (5,8\%) leve restrição e somente 1 (1,4\%) apresentou grande restrição. Em relação à escala SALSA, o escore variou de 19 a 64 pontos. Os escores mais prevalentes foram 20 pontos, registrados por $16(23,2 \%)$ pessoas, e 19 pontos observados em $9(13 \%)$ participantes. Somente $1(1,4 \%)$ participante pontuou escore acima de 50 . 0 escore de consciência de risco variou de 0 a 7 , sendo que 39 (56,5\%) pontuaram 0 e somente $1(1,4 \%)$ pontuou 7 . A população do estudo era composta principalmente por mulheres, não refletindo os dados nacionais e da literatura. ${ }^{17}$

Um ponto importante da escala SALSA, observado no escore de consciência de risco, é a avaliação do quanto o paciente tem ciência de suas limitações e dos riscos para realizar certas atividades. O resultado desse escore facilita a identificação de quais ações devem ser trabalhadas com o paciente para prevenção de incapacidades, sendo sugestivo para as atividades do grupo de autocuidado. ${ }^{14}$

Nesse sentido, o uso das escalas SALSA e de Participação Social no serviço de saúde podem nortear o cuidado ao paciente com hanseníase, pois a partir dessas escalas 
os profissionais podem compreender os fatores que interferem e são determinantes no processo de adoecimento do paciente. ${ }^{4}$

Na correlação entre SALSA e Participação Social, Barbosa et al. ${ }^{17}$ verificaram que os pacientes classificados como sem restrição à participação social estavam divididos em quase todos os escores da escala SALSA. O indivíduo que apresentou extrema restrição à participação obteve 23 pontos na escala SALSA, ou seja, não teve limitação de atividades. Resultado condizente com o presente estudo, porém com uma amostra muito maior de pacientes. $\mathrm{A}$ aplicação das escalas permite uma abordagem também do fator emocional, possibilitando identificar necessidades para acompanhamento individual ou em grupo. Pesquisa que questionou cinco pacientes em tratamento para hanseníase se continuavam participando das mesmas atividades após o diagnóstico teve como resultado que três pacientes referiram mudanças na participação social em razão de dor e sequelas físicas ou emocionais. ${ }^{31}$

Em relação ao grupo de autocuidado realizado na pesquisa, constatou-se que os escores da escala SALSA e de Participação Social tiveram uma melhora após a quarta participação no grupo, o que ressalta sua relevância na melhora da qualidade de vida dos pacientes com hanseníase. O grupo foi desenvolvido com estratégias de fácil entendimento e linguagem simples, com discussões de temas e verbalização de experiências vivenciadas. A troca entre os participantes e os profissionais envolvidos proporcionou aos pacientes conhecer a hanseníase e desenvolver habilidades para o autocuidado.

Embora a equipe multiprofissional participasse das reuniões quando era convidada para abordar tema relativo à sua área de atuação, não se percebeu a corresponsabilização dos mesmos com o grupo, uma vez que não participaram do planejamento e organização das reuniões.

É importante a criação de espaços de acolhimento e escuta para os pacientes expressarem seus sentimentos e falarem da doença. Além disso, os profissionais precisam utilizar linguagem clara e adequada ao perfil dos pacientes a fim de que as informações sejam assimiladas. ${ }^{32}$ As atividades educativas são vistas como essenciais no processo terapêutico do indivíduo. O profissional precisa se atentar aos variados significados que a experiência da hanseníase traz para as pessoas acometidas e que a atividade educativa é um elo com o paciente. ${ }^{33}$ Dessa forma, o intuito da educação em saúde é favorecer a qualidade de vida do paciente, contribuir para que este repense suas atitudes e viva mais saudável, bem como permitir que esses pacientes troquem conhecimentos e experiências, aumentando assim suas habilidades de responder às condições de saúde. ${ }^{34}$ Evidenciou-se na fala dos entrevistados neste estudo o reconhecimento quanto aos benefícios obtidos pela participação no grupo de autocuidado. De forma semelhante, pesquisa que envolveu 20 usuários em tratamento para hanseníase na Zona da Mata Mineira mostrou que os pacientes que participavam de grupos de apoio relataram benefícios pela troca de experiências, conhecimento sobre a doença e orientações de autocuidado que os profissionais de saúde proporcionavam. ${ }^{35}$ 
Outro estudo identificou que os pacientes compreenderam a importância do grupo de autocuidado como ferramenta de informação, local de escuta e de aprendizado em saúde, bem como instrumento de promoção da adesão ao tratamento e de minimização do preconceito. ${ }^{36}$

Nesse mesmo sentido, uma pesquisa com objetivo de avaliar o conhecimento adquirido sobre prevenção de incapacidades no controle da hanseníase, pelos participantes de um grupo de autocuidado em um hospital, constatou que participar das reuniões do grupo melhorou bastante o conhecimento sobre autocuidado. ${ }^{34} \mathrm{Um}$ estudo realizado em Moçambique com o objetivo de compreender as percepções das pessoas com hanseniase em relação aos beneficios e desafios dos grupos de autocuidado mostrou que a maioria dos entrevistados estavam satisfeitos em participar dos grupos e obtiveram melhor compreensão sobre as incapacidades e feridas, autonomia para lutar pelos seus direitos, socialização e criação de vínculos, oportunidade para sair de casa, entre outros. ${ }^{37}$

A doença muda o cotidiano do paciente devido a alterações em seu estilo de vida. As reações quanto ao diagnóstico e tratamento e a forma de entender as situações são diferentes para cada pessoa. Assim, o paciente precisa ser orientado a se autoconhecer, ser capaz de escolher a maneira de viver após o diagnóstico e direcionar seu autocuidado, não apenas ser instruído para prática tradicional de tratamento. ${ }^{8}$

Identificaram-se muitas justificativas dos pacientes para não participarem do grupo de autocuidado, mas, embora não expressado na maioria das falas, percebeu-se que muitos não participaram por não desejarem se expor à comunidade devido ao estigma e preconceito que a doença ainda carrega. Em pesquisa realizada por Pelizzari et al. ${ }^{38}$, os pacientes relataram sentir vergonha ao falar que tinham hanseníase, seja em virtude do medo da doença pela outra pessoa ou pelo receio de preconceito. Os entrevistados referiram conviver com estigma e preconceito. Receber o diagnóstico para eles foi motivo de angústia e resistência, visto que não esperavam ter a doença. Um impasse para os pacientes de hanseníase consiste em contar ou não o diagnóstico para outras pessoas, isso ressalta o preconceito que as pessoas ainda apresentam e o autopreconceito. Nesse sentido, evidenciam-se concepções negativas que podem ser resultado da falta de conhecimento sobre a hanseníase ou do estigma milenar ainda presente, que pode os levar ao isolamento.39

Pesquisa desenvolvida na Índia sobre perfil social e psicológico de 251 individuos com hanseníase demonstrou que a maioria $(88,4 \%)$ dos pacientes não sofreu atitude negativa de outras pessoas que sabiam de seu diagnóstico, entretanto, praticamente todos tiveram reação de luto e medo de sofrerem discriminação no ambiente social quando receberam o diagnóstico. Quase metade referiu sentir medo de ser taxado de pecador e $14 \%$ foram indiferentes e trataram a hanseníase como qualquer outra doença. Alguns pacientes evitaram participar de reuniões sociais após o diagnóstico. ${ }^{40}$ 
Loures et al. ${ }^{35}$ demonstraram o desconhecimento dos pacientes sobre a doença, a importância que estes davam à cura pelo receio de transmitir aos familiares e a relação de apoio em que a família foi a maior fonte de suporte. A participação social não teve alteração, mas alguns pacientes referiram ter se afastado do convívio social por várias razões, não obrigatoriamente devido à hanseníase.

O suporte social é uma fonte de proteção, conforme modelo biopsicossocial de saúde, em que ouvir o indivíduo é primordial e o cuidado não deve ser focado apenas na doença, mas em todo o contexto social em que esse indivíduo está situado. ${ }^{28}$ Os familiares são importantes no acompanhamento e enfrentamento da hanseníase por conviverem e estarem próximos dos pacientes. ${ }^{38}$ Dessa forma, o suporte dos profissionais, familiares e amigos é importante para que o paciente tenha autoestima, fortaleça seus vínculos e tenha participação plena da vida. ${ }^{31}$

Ademais, é necessário enfatizar que o indivíduo deve ser considerado em sua totalidade. Os comprometimentos da hanseníase são muito mais que físicos, passam do biológico e material e atingem o psicossocial, necessitando, assim, de uma atenção individual e especializada. ${ }^{9}$

\section{CONCLUSÃO}

Constatou-se que a adesão ao grupo pelos pacientes em tratamento ainda é baixa e as justificativas por não comparecer são muitas. Portanto, torna-se necessário continuar a análise das causas da não adesão e o planejamento de estratégias para maior participação. Uma equipe multiprofissional mais empenhada no grupo de autocuidado e o convite às famílias dos pacientes para participarem do grupo podem favorecer a adesão desses pacientes, assim como diminuir o estigma e o preconceito com a doença.

Por outro lado, os pacientes que participaram do grupo relataram que os encontros são muito importantes e se sentiram mais confiantes para lidar com a doença e o tratamento. Dessa forma, os grupos de autocuidado podem auxiliar na prevenção das consequências da hanseníase, ser fonte de suporte aos pacientes para enfrentarem melhor a doença e melhorarem a qualidade de suas vidas.

A limitação de atividades e a participação social dos pacientes podem ser prejudicadas devido à hanseníase, no entanto, o grupo de autocuidado contribui favoravelmente para que a interferência da doença na vida diária do indivíduo seja mais amena.

Destaca-se como limitações deste estudo a reduzida amostra devido à realidade própria do centro de saúde, com um número relativamente pequeno de pacientes acompanhados durante o período de coleta de dados e que estivessem dentro dos critérios de inclusão/exclusão do estudo. No entanto, além da contribuição científica e a potencialidade de somar conhecimento, o estudo tem 
papel social na busca pela diminuição do estigma da doença e inclusão social dos pacientes a partir da prevenção de incapacidades e de limitação de atividades através do autocuidado.

Sugere-se que sejam realizadas novas pesquisas com uma amostra maior para descrição de como a hanseníase interfere no dia a dia do paciente em relação à limitação de atividades e participação social.

\section{REFERÊNCIAS}

1. ORGANIZACIÓN MUNDIAL DE LA SALUD. Centro de prensa. Lepra. Ginebra: OMS. 2019 [Citado em 2019 mar 16]. Disponível em: https://www.who.int/es/news-room/ fact-sheets/detail/leprosy.

2. WORLD HEALTH ORGANIZATION. Global Health Observatory data repository. Leprosy-Number of new leprosy cases. Data by country. Geneva: WHO. 2018 [Citado em 2018 dez 28]. Disponível em: http://apps.who.int/gho/data/view.main.95300.

3. DATASUS (Brasil). Informações de Saúde (Tabnet). Epidemiológicas e morbidade. Casos de Hanseníase (SINAN). Hanseníase. Geral-indicadores de 2017. Brasil: Ministério da Saúde. 2018 [Citado em 2019 jan 18]. Disponível em: http://tabnet. datasus.gov.br/cgi/dhdat.exe?hanseniase/hantfbr17.def.

4. Lima IB, Simpson CA, Cabral AMF. Limitação de atividades e participação social em pacientes com hanseníase. Rev enferm UFPE on line. 2014 abr; 8(4):994-1001.

5. Duarte MTC, Ayres JA, Simonetti JP. Consulta de enfermagem: estratégia de cuidado ao portador de hanseníase em atenção primária. Texto Contexto Enferm. 2009 janmar; 18(1): 100-7.

6. Girardi DR, Takahara AL. Autoestima em portadores de hanseníase e diabetes: Variáveis das escalas salsa e participação com o apoio das Redes bayesianas. FAE Centro Universitário, Núcleo de Pesquisa Acadêmica - NPA. Programa de Apoio à Iniciação Científica - PAIC 2013-2014. Disponível em: https://cadernopaic.fae.edu/ cadernopaic/article/viewFile/76/75.

7. Batista TVG, Vieira CSCA, Paula MAB. A imagem corporal nas ações educativas em autocuidado para pessoas que tiveram hanseníase. PhysisRevista de Saúde Coletiva. 2014; 24(1):89-104.

8. Souza IA, Ayres JA, Meneguin S, Spagnolo RS. Autocuidado na percepção de pessoas com hanseníase sob a ótica da complexidade. Esc Anna Nery, Rev de Enferm. 2014 jul-set; 18(3):510-4.

9. Galan NGA, Beluci ML, Marciano LHSC, Prado RBR, Oliveira NGG, Bonini AG, et al. Avaliação da prática do autocuidado domiciliar em hanseníase. Hansen Int. 2014; 39(2):27-35. 
10. Ministério da Saúde (Brasil). Secretaria de Vigilância em Saúde, Departamento de Vigilância Epidemiológica. Guia de apoio para grupos de autocuidado em hanseníase. Brasília: Ministério da Saúde; 2010a.

11. Ministério da Saúde (Brasil). Secretaria de Vigilância em Saúde, Departamento de Vigilância Epidemiológica. Manual de prevenção de incapacidades. Brasília: Ministério da Saúde; 2008.

12. Ministério da Saúde (Brasil). Secretaria de Vigilância em Saúde, Departamento de Vigilância Epidemiológica. Capacitação em prevenção de incapacidades em hanseníase: caderno do monitor. Brasília: Ministério da Saúde; 2010b.

13. SALSA SCALE. Users Manual. Version 1.1. Date of last revision: 21st July 2010. Disponível em: https://www.infontd.org/files/SALSA\%20manual\%20v1.1pdf_0.pdf.

14. Oliveira LR, Nascimento AR, Nascimento MMP, Pereira AP, Lemos ICS, Kerntopf MR. Limitação de atividades e participação social entre usuários de um grupo de autocuidado em hanseníase. R. Interd. 2016 jan-mar; 9(1):171-81.

15. PARTICIPATION SCALE. Users Manual. Version 6.0. Date of last revision: 16st. April 2010. Disponível em: https://www.infontd.org/files/Participation\%20Scale\%20 Users\%20Manual\%20v.\%206.0_1.pdf.

16. INFOLEP. Leprosy Information Services. Participation Scale. Disponível em: https:// www.leprosy-information.org/content/participation-scale.

17. Barbosa JC, Ramos Jr AN, Alencar MJF, Castro CGJ. Pós-alta em Hanseníase no Ceará: limitação da atividade funcional, consciência de risco e participação social. Rev Bras Enferm. 2008; 61(esp):727-33.

18. Pádua EMM. Metodologia da pesquisa: abordagem teórica-prática. 10a edição. Campinas: Papirus; 2004.

19. Ministério da Saúde (Brasil). Secretaria de Vigilância em Saúde. Departamento de Vigilância Epidemiológica. Autocuidado em hanseníase: face, mãos e pés. Brasília: Ministério da Saúde; 2010.

20. Ministério da Saúde (Brasil). Secretaria de Vigilância em Saúde. Departamento de Vigilância Epidemiológica. Eu me cuido e vivo melhor. Brasília: Ministério da Saúde; 2010.

21. Bardin L. Análise de Conteúdo. Lisboa: Persona Edições; 2011.

22. Ministério da Saúde (Brasil). Conselho Nacional de Saúde. Resolução no 466 de 12 de dezembro de 2012. Disponível em: http://bvsms.saude.gov.br/bvs/saudelegis/ cns/2013/res0466_12_12_2012.html.

23. Gaudenci EM, Nardelli GG, Almeida Neto OP, Malaquias BSS, Carvalho BT, Pedrosa LAK. Qualidade de vida, sintomas depressivos e incapacidade física de pacientes com hanseníase. Hansen Int. 2015; 40(2):48-58. 
24. Palú FH, Cetolin SF. Perfil clínico-epidemiológico dos pacientes com hanseníase no extremo oeste catarinense, 2004 a 2014. Arq. Catarin Med. 2015 abrjun; 44(2):90-8.

25. Moura ADA, Albuquerque ERO, Chaves ES, Souza AR, Lima GG, Chaves CS. Perfil dos portadores de hanseníase de um centro de referência de um estado brasileiro. Revenferm UERJ. 2016; 24(6).

26. Ministério da Saúde (Brasil). Secretaria de Vigilância em Saúde, Departamento de Vigilância das Doenças Transmissíveis. Guia prático sobre a hanseníase. Brasília: Ministério da Saúde; 2017.

27. Silva SF, Griep RH. Reação hansênica em pacientes portadores de hanseníase em centros de saúde da Área de Planejamento 3.2. do Município do Rio de Janeiro. Hansen Int. 2007; 32(2):155-62.

28. Loures LF, Mármora CHC. Suporte e participação social em indivíduos com hanseníase. O Mundo da Saúde. 2017; 41(2):244-52.

29. Brakel WHV, Sihombing B, Djarir H, Beise K, Kusumawardhani L, Yulihane R, et al. Disability in people affected by leprosy: the role of impairment, activity, social participation, stigma and discrimination. Glob Health Action. 2012. Disponível em: https://www.tandfonline.com/doi/full/10.3402/gha.v5i0.18394.

30. Ikehara E, Nardi SMT, Ferrigno ISV, Pedro HSP, Paschoal VDA. Escala Salsa e grau de Incapacidades da Organização Mundial de Saúde: avaliação da limitação de atividades e deficiência na hanseníase. Actafisiatr. 2010; 17(4):169-74.

31. Marinho FD, Macedo DCF, Sime MM, Paschoal VDA, Nardi SMT. Percepções e sentimentos diante do diagnóstico, preconceito e participação social de pessoas acometidas pela hanseníase. Arq. Ciênc. Saúde. 2014 jul-set; 21(3):46-52.

32. Nunes JM, Oliveira EM, Vieira NFC. Hanseníase: conhecimentos e mudanças na vida das pessoas acometidas. Ciência \& Saúde Coletiva. 2011; 16(Supl. 1):1311-8.

33. Silva MCD, Paz EPA. Educação em saúde no programa de controle da hanseníase: a vivência da equipe multiprofissional. Esc Anna Nery, Rev Enferm. 2010 abr-jun; 14(2):223-9.

34. Pinheiro MGC, Silva SYB, Silva FS, Ataide CAV, Lima IB, Simpson CA. Conhecimento sobre prevenção de incapacidades em um grupo de autocuidado em hanseníase. REME, Rev Min Enferm. 2014 out-dez; 18(4):901-6.

35. Loures LF, Mármora CHC, Barreto J, Duppre NC. Percepção do estigma e repercussões sociais em indivíduos com hanseníase. Psicologia em Estudo. 2016 out-dez; 21(4):665-75.

36. Duarte LMCPS, Simpson CA, Silva TMS, Moura IBL, Isoldi DMR.Ações de autocuidado de pessoas com hanseníase. Rev enferm UFPE on line. 2014 ago; 8(8):2816-22. 
37. Deepak S, Hansine PE, Braccini C. Self-care groups of leprosy-affected people in Mozambique. Lepr Rev. 2013; 84:283-91.

38. Pelizzari VDZV, Arruda GO, Marcon SS, Fernandes CAM.Percepções de pessoas com hanseníase acerca da doença e tratamento. Rev Rene. 2016 jul-ago; 17(4):466-74.

39. Carneiro DF, Silva MMB, Pinheiro M, Palmeira IP, Matos EVM, Ferreira AMR. Itinerários terapêuticos em busca do diagnóstico e tratamento da hanseníase. Rev. baiana enferm. 2017; 31(2).

40. Kumar S, Pandey SS, Kaur P. Psychosocial consequences of leprosy patients in eastern up. Indian J. Prev. Soc. Med. 2014; 45(1-2). 